\title{
Constrained LQR for Low-Precision Data Representation *
}

\author{
Stefano Longo $^{\text {a }}$, Eric C. Kerrigan ${ }^{\text {b,c }}$, George A. Constantinides ${ }^{\text {b }}$ \\ ${ }^{a}$ Department of Automotive Engineering, Cranfield University, Cranfield, MK43 OAL, UK \\ ${ }^{\mathrm{b}}$ Department of Electrical and Electronic Engineering, Imperial College London, London, SW7 2AZ, UK \\ ${ }^{\mathrm{c}}$ Department of Aeronautics, Imperial College London, London, SW7 2AZ, UK
}

\begin{abstract}
Performing computations with a low-bit number representation results in a faster implementation that uses less silicon, and hence allows an algorithm to be implemented in smaller and cheaper processors without loss of performance. We propose a novel formulation to efficiently exploit the low (or non-standard) precision number representation of some computer architectures when computing the solution to constrained LQR problems, such as those that arise in predictive control. The main idea is to include suitably-defined decision variables in the quadratic program, in addition to the states and the inputs, to allow for smaller roundoff errors in the solver. This enables one to trade off the number of bits used for data representation against speed and/or hardware resources, so that smaller numerical errors can be achieved for the same number of bits (same silicon area). Because of data dependencies, the algorithm complexity, in terms of computation time and hardware resources, does not necessarily increase despite the larger number of decision variables. Examples show that a 10-fold reduction in hardware resources is possible compared to using double precision floating point, without loss of closed-loop performance.
\end{abstract}

Key words: Embedded systems, Control of constrained systems, Predictive control, Optimization, Number representation

\section{Introduction}

It is common practice to optimize the performance of sophisticated algorithms, like online model-based optimization methods, only at a high level of abstraction, viewing the implementation as a different, decoupled problem [5]. This practice might no longer be reasonable due to the fact that the high degree of flexibility of new computer architectures remains largely underexploited [2]. The standard doubleor single-precision floating-point representation may be unnecessarily precise for an application where such precision would be better traded for more important aspects. For a control algorithm, these aspects could be computational speed, processor cost and reduced power consumption. To motivate this point further, we have plotted in Fig. 1 the resources needed on a Virtex-6 FPGA for an implementation of the predictive control algorithm of [10]. Working in single precision (23 bits), rather than double (52 bits), already gives a substantial improvement, but reducing the number of mantissa bits even further allows one to reduce the total hardware resources by one order of magnitude and do the computations almost twice as fast. The ability to reliably run an optimization algorithm in a low precision platform is also

\footnotetext{
ऋ This research has been supported by the EPSRC (EP/G031576/1, EP/F041004/1) and FP7/2007-2013 (FP7-ICT2009-4 248940). Part of this work has been presented at the IFAC NMPC'12 conference, the Netherlands, and ECC'13, Switzerland. Corresponding author: S. Longo, tel.: +44 (0)1234 758581.

Email addresses: s. longodcranfield.ac.uk (Stefano Longo), e.kerriganeimperial.ac.uk

(Eric C. Kerrigan), g.constantinides dimperial.ac.uk (George A. Constantinides).
}

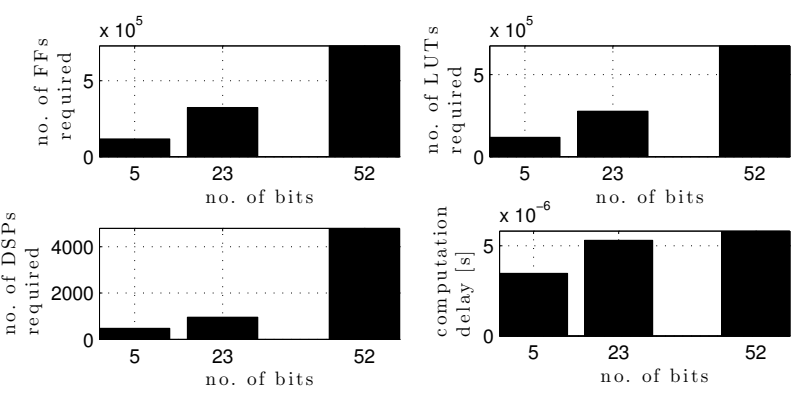

Fig. 1. FPGA resources required for double ( 52 bits), single (23 bits) and custom (5 bits) precision for data representation of a model predictive control algorithm (6 states, 2 inputs and horizon length of 200 steps). Reducing the number of bits (only the mantissa bits are considered here) used for data representation significantly reduces the hardware resources (Flip-Flops, Look-Up-Tables, Digital-Signal-Processing units) required and the algorithm computational delay (calculated at a clock frequency of $200 \mathrm{MHz}$ and assuming 10 interior-point iterations and a fully parallel implementation).

motivated by the fact that most microprocessors in embedded systems do not offer any support for double precision floating-point or for floating-point at all [5].

In view of the above, we propose a new formulation for the Quadratic Programming (QP) problem (resulting from the constrained LQR problem) that allows one to exploit low precision number representations efficiently [7]. This formulation is mathematically equivalent to the non-condensed formulation where inputs and states appear as decision variables [10]. The difference here is that we represent the continuous-time plant model (used for the prediction) in the 
so-called delta (or incremental) form [3,4,8,9], and include additional decision variables. The delta form has the virtue of separating the important information stored in the system's transition matrix from quantities of non-comparable size. By doing so, the truncation due to finite precision arithmetic becomes less detrimental than for the more commonly used shift form; hence, the algorithm will be numerically more stable. The modern need for the implementation of fast constrained LQ solvers in inexpensive hardware [11] justifies this hardware-algorithm co-design approach and makes it necessary to revive the use of discretization methods that are numerically more stable.

It will be shown that, for some examples, it is possible to achieve good closed-loop performance with only 5 bits for the mantissa, which translates into fewer hardware resources needed, faster computation and less power consumption. More interestingly, it will be shown that, even with the same number of bits for data representation and despite the number of decision variables in the QP becoming larger, i) the computational cost of solving this problem still scales linearly with the horizon length, ii) the data dependencies of the algorithm are such that it can be implemented at essentially no extra hardware costs (hardware adders and multipliers are reused efficiently) and iii) the algorithm is at least as fast as its shift equivalent. In fact, the algorithm in delta domain is normally faster because the better numerical stability leads to fewer interior-point iterations when an interior-point method is used, i.e. one has faster convergence to the solution.

\section{Constrained LQR problem formulation in delta do- main}

Consider the continuous-time LTI plant model

$$
\dot{x}(t)=A_{c} x(t)+B_{c} u(t),
$$

where $x(t) \in \mathbb{R}^{n_{x}}, u(t) \in \mathbb{R}^{n_{u}}$ and $\left(A_{c}, B_{c}\right)$ is a stabilizable pair. The control input $u(\cdot)$ is a signal created by a sampleand-hold element for a sampling period $h$ such that $u(t)=$ $u(i h)$ for all $t \in[i h, i h+h)$ and $i \in \mathbb{N}_{0}$ is the sampling instant. The solution of the sampled-data model is given by

$$
x(i h+h)=\underbrace{e^{A_{c} h}}_{\triangleq A_{s}} x(i h)+\underbrace{\left[\int_{0}^{h} e^{A_{c}(h-\tau)} B_{c} d \tau\right]}_{\triangleq B_{s}} u(i h),
$$

where $i$ denotes the sampling instant and the matrix exponential $e^{A_{c} h}$ is defined by the matrix series

$$
e^{A_{c} h} \triangleq I+A_{c} h+\frac{\left(A_{c} h\right)^{2}}{2 !}+\frac{\left(A_{c} h\right)^{3}}{3 !}+\ldots
$$

If the product $A_{c} h$ in (3) results in a matrix with entries much smaller than one, the transition matrix $A_{s}$ in (2) will be a matrix where the elements on the diagonal are the summation of 1 with a much smaller number. These coefficients have to be stored in a computer with finite word length. In finite arithmetic, the coefficients will be truncated, hence some of the information contained in $A_{c} h+\frac{\left(A_{c} h\right)^{2}}{2 !}+\frac{\left(A_{c} h\right)^{3}}{3 !}+\ldots$
- which is where the plant dynamics are represented - will be lost. This numerical problem can be ameliorated by substituting (3) into (2) and rewriting it as

$$
\begin{aligned}
x(i h+h) & =x(i h)+h \underbrace{\left[A_{c}+\frac{A_{c}^{2} h}{2 !}+\frac{A_{c}^{3} h^{2}}{3 !}+\ldots\right]}_{\triangleq A_{\delta}} x(i h) \\
+ & h \underbrace{\left[B_{c}+\frac{A_{c} h B_{c}}{2 !}+\frac{A_{c}^{2} h^{2} B_{c}}{3 !}+\ldots\right]}_{\triangleq B_{\delta}} u(i h),(4)
\end{aligned}
$$

which is mathematically equivalent to (2). However, separating the identity matrix from the summation tail — defined as $A_{\delta}$ in (4) - has the effect of reducing the numerical errors in a finite precision floating-point representation. These matrices can be computed as $A_{\delta}=\Omega A_{c}$ and $B_{\delta}=\Omega B_{c}$, where

$$
\Omega \triangleq \frac{1}{h} \int_{0}^{h} e^{A_{c} \tau} d \tau=\frac{1}{h}\left[\begin{array}{ll}
I & 0
\end{array}\right] \exp \left[\begin{array}{cc}
h A_{c} & h I \\
0 & 0
\end{array}\right]\left[\begin{array}{l}
0 \\
I
\end{array}\right] .
$$

By adopting the more convenient notation $x(i h) \triangleq x_{i}$ (and similarly for other vectors) and introducing a new vector $\delta_{i} \in$ $\mathbb{R}^{n_{x}}$, we can rewrite (4) as

$$
\begin{array}{r}
\delta_{i}=A_{\delta} x_{i}+B_{\delta} u_{i}, \\
x_{i+1}=x_{i}+h \delta_{i},
\end{array}
$$

which is in fact the delta (or incremental) form of (1) $[3,4$, $8,9]$, an alternative to the commonly used shift form of (2). Associated with the system in (6), consider the discrete-time finite-horizon LQ problem defined by the cost function

$$
V(\theta) \triangleq x_{N}^{\prime} Q_{f} x_{N}+\sum_{k=0}^{N-1}\left[\begin{array}{l}
x_{k} \\
u_{k}
\end{array}\right]^{\prime}\left[\begin{array}{cr}
Q & M \\
M^{\prime} & R
\end{array}\right]\left[\begin{array}{l}
x_{k} \\
u_{k}
\end{array}\right]
$$

where $N$ is the number of samples for the prediction horizon, $\left[\begin{array}{cc}Q & M \\ M^{\prime} & R\end{array}\right] \geq 0, Q=Q^{\prime} \geq 0, R=R^{\prime}>0, Q_{f}=Q_{f}^{\prime}>0$ and $\theta$ is defined below. These matrices are given to define a controller for an ideal closed-loop performance of the sample-data system. Alternatively, they can be computed via discretization of a continuous-time LQ problem [6], [1, pp. 411-412]. For the constrained LQR problem we assume full state feedback and we suppose that constraints exist on the input moves and on the states; in order to solve the QP problem, we assume that the constraints lie in a polyhedral set, i.e. we can write them as $J x_{k}+E u_{k} \leq d$. Given a measurement or estimate of the current state $\hat{x} \triangleq x_{0}$ and an input sequence $\left(u_{0}, u_{1}, \ldots, u_{N-1}\right)$, let $x_{k}, k \in \mathbb{N}_{0}$, be the predicted state after $k$ samples. The optimal control problem to solve is 


$$
\begin{array}{ll}
\min _{\theta} & \frac{1}{2} V(\theta) \\
\text { s.t. } & \delta_{k}=A_{\delta} x_{k}+B_{\delta} u_{k}, \quad x_{0}=\hat{x} \\
& x_{k+1}=x_{k}+h \delta_{k} \\
& J x_{k}+E u_{k} \leq d \\
& \forall k \in\{0,1, \ldots, N-1\} .
\end{array}
$$

Problem (8) can be written as a QP problem of the form

$$
\min _{\theta} \frac{1}{2} \theta^{\prime} H \theta+\theta^{\prime} c \quad \text { s.t. } \quad F \theta=f, G \theta \leq g
$$

where matrices and vectors depend on the choice of the vector of decision variables $\theta$. Although we generally only wish to find the sequence of control inputs $\left(u_{0}, u_{1}, \ldots, u_{N-1}\right)$, it has been shown $[10,12]$ that including the states $\left(x_{1}, x_{2}, \ldots, x_{N}\right)$ in $\theta$ results in the $\mathrm{QP}$ matrices having a particular structure, which is desirable for fast online optimization. In our formulation, we not only include the states as decision variables, but also the auxiliary variables $\left(\delta_{0}, \delta_{1}, \ldots, \delta_{N-1}\right)$. Thus, we let

$$
\theta \triangleq\left[\begin{array}{llllllll}
u_{0}^{\prime} & \delta_{0}^{\prime} & x_{1}^{\prime} & u_{1}^{\prime} & \delta_{1}^{\prime} & x_{2}^{\prime} & \ldots & x_{N}^{\prime}
\end{array}\right]^{\prime}, \theta \in \mathbb{R}^{N\left(n_{u}+2 n_{x}\right)}
$$

which results in the following matrices for the QP in (9):

$$
\begin{aligned}
& H \triangleq\left[\begin{array}{cccccc}
R & & & & & \\
& 0 & & & & \\
& & Q & M & & \\
& M^{\prime} & R & & \\
& & & \ddots & \\
& & & & & Q_{f}
\end{array}\right], \quad c=\left[\begin{array}{c}
0 \\
0 \\
\vdots \\
0
\end{array}\right],
\end{aligned}
$$

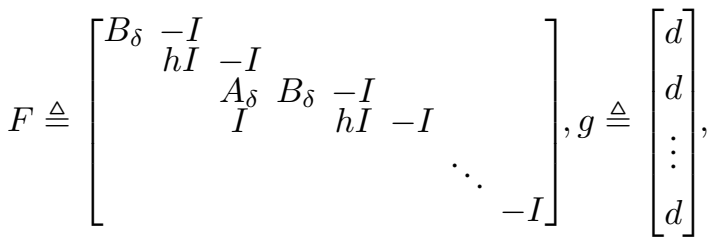

$$
\begin{aligned}
& G \triangleq\left[\begin{array}{ccccccccc}
E & 0 & & & & & & & \\
& J & E & 0 & & & & & \\
& & & & J & E & 0 & & \\
& & & & & & \ddots & \\
& & & & & & & J
\end{array}\right], f \triangleq\left[\begin{array}{c}
-A_{\delta} \hat{x} \\
-\hat{x} \\
0 \\
\vdots \\
\end{array}\right.
\end{aligned}
$$

Note that the QP (9) can be solved in low precision using solvers other than the one we will present in Section 3; however, if $\delta_{k}$ is not included in $\theta$, then no solver will be able to recover the 'lost' information contained in $A_{\delta}$.

\section{Solution of the QP by an interior-point method with block elimination}

Since the QP in (9) has to be solved in an embedded platform with limited computational power, it might be preferable to compute the solution using an interior-point method so that the algorithm works with matrices with a fixed structure (unlike active-set methods). In particular, we will consider a well-established primal-dual interior-point method with infeasible start [10]. The equations derived in this section are analogous to the ones presented in [10], which were derived for a vector of decision variables containing the sequence of future inputs and states only, using the shift form. Here, by also including the $\delta_{k}$ as decision variables, one can formulate an equivalent algorithm in delta domain. Because of space consideration, we skip all the details and point out that the most computationally expensive part of the algorithm is the solution of the linear systems for the calculation of the search direction. By exploiting the banded structure of the Hessian and the constraint matrix, we can construct a system of linear equations, equivalent to the one in [10, eq. 38]:

$$
\mathcal{A} \xi=\beta,
$$

where

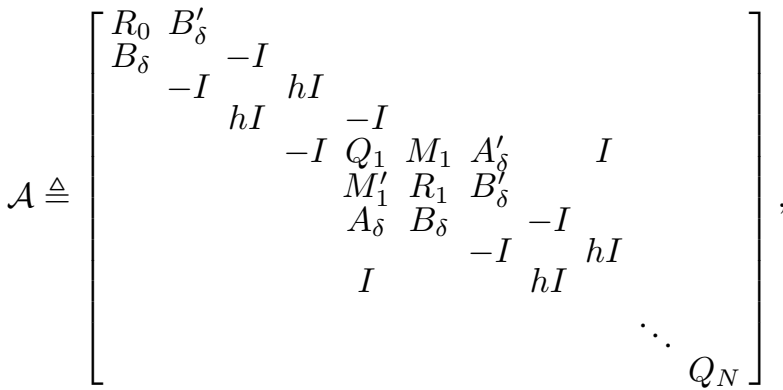

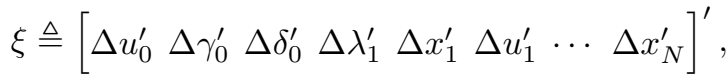

$$
\begin{aligned}
& \beta \triangleq\left[\left(r_{0}^{u}\right)^{\prime}\left(r_{0}^{\gamma}\right)^{\prime}\left(r_{0}^{\delta}\right)^{\prime}\left(r_{1}^{\lambda}\right)^{\prime}\left(r_{1}^{x}\right)^{\prime}\left(r_{1}^{u}\right)^{\prime} \cdots\left(r_{N}^{x}\right)^{\prime}\right]^{\prime} \text {, }
\end{aligned}
$$

and $R_{k}, Q_{k}, M_{k}, r_{k}^{u}, r_{k}^{\gamma}, r_{k}^{\delta}, r_{k}^{\lambda}, r_{k}^{x}$, for all $k$, can be derived following the same procedure as in [10]. If we let $P_{k} \triangleq Q_{k}$ and $p_{k} \triangleq r_{k}^{x}$ and perform a series of block eliminations in (12), we get

$$
\begin{aligned}
\Delta u_{0} & =\left(R_{0}+h^{2} B_{\delta}^{\prime} P_{1} B_{\delta}\right)^{-1}\left(h^{2} B_{\delta}^{\prime} P_{1} r_{0}^{\gamma}+h B_{\delta}^{\prime} P_{1} r_{1}^{\lambda}\right. \\
& \left.+h B_{\delta}^{\prime} p_{1}+B_{\delta}^{\prime} r_{0}^{\delta}+r_{0}^{u}\right) \\
\Delta \gamma_{0} & =h^{2} P_{1} B_{\delta} \Delta u_{0}-h^{2} P r_{0}^{\gamma}-h P_{1} r_{1}^{\lambda}-h p_{1}-r_{0}^{\delta} \\
\Delta \delta_{0} & =B_{\delta} \Delta u_{0}-r_{0}^{\gamma} \\
\Delta \lambda_{1} & =h P \Delta \delta_{0}-P_{1} r_{1}^{\lambda}-p_{1} \\
\Delta x_{1} & =h \Delta \delta_{0}-r_{1}^{\lambda}
\end{aligned}
$$

and

$$
\begin{aligned}
\Delta u_{k-1} & =\left(R_{k-1}+h^{2} B_{\delta}^{\prime} P_{k} B_{\delta}\right)^{-1}\left(\left(r_{k-1}^{u}+h^{2} B_{\delta}^{\prime} P_{k} r_{k-1}^{\gamma}\right.\right. \\
& \left.+B_{\delta}^{\prime} r_{k-1}^{\delta}+h B_{\delta}^{\prime} P_{k} r_{k}^{\lambda}+h B_{\delta}^{\prime} p_{k}\right) \\
& \left.-\left(M_{k-1}^{\prime}+h^{2} B_{\delta}^{\prime} P_{k} A_{\delta}+h B_{\delta}^{\prime} P_{k}\right) \Delta x_{k-1}\right), \\
\Delta \gamma_{k-1} & =h^{2} P_{k}\left(B_{\delta} \Delta u_{k-1}+A_{\delta} \Delta x_{k-1}-r_{k-1}^{\gamma}\right) \\
& -h P_{k}\left(\Delta x_{k-1}-r_{k}^{\lambda}\right)-h p_{k}-r_{k-1}^{\delta}, \\
\Delta \delta_{k-1} & =A_{\delta} \Delta x_{k-1}+B_{\delta} \Delta u_{k-1}-r_{k-1}^{\gamma}, \\
\Delta \lambda_{k} & =p_{k-1}-P_{k-1} \Delta x_{k-1}, \\
\Delta x_{k} & =\Delta x_{k-1}+h \Delta \delta_{k-1}-r_{k}^{\lambda},
\end{aligned}
$$

where 


$$
\begin{aligned}
P_{k-1} & \triangleq Q_{k-1}+P_{k}+h^{2} A_{\delta}^{\prime} P_{k} A_{\delta}+h A_{\delta}^{\prime} P_{k}+h P_{k} A_{\delta} \\
& -\left(M_{k-1}+h^{2} A_{\delta}^{\prime} P_{k} B_{\delta}+h P_{k} B_{\delta}\right)\left(R_{k-1}\right. \\
& \left.+h^{2} B_{\delta}^{\prime} P_{k} B_{\delta}\right)^{-1}\left(M_{k-1}^{\prime}+h^{2} B_{\delta}^{\prime} P_{k} A_{\delta}+h B_{\delta}^{\prime} P_{k}\right)
\end{aligned}
$$

$$
\begin{aligned}
p_{k-1} & \triangleq r_{k-1}^{x}+P_{k} r_{k}^{\lambda}+p_{k}+h^{2} A_{\delta}^{\prime} P_{k} r_{k-1}^{\gamma}+A_{\delta}^{\prime} r_{k-1}^{\delta} \\
& +h A_{\delta}^{\prime} P_{k} r_{k}^{\lambda}+h A_{\delta}^{\prime} p_{k}+h P_{k} r_{k-1}^{\gamma}-\left(M_{k-1}\right. \\
& \left.+h^{2} A_{\delta}^{\prime} P_{k} B_{\delta}+h P_{k} B_{\delta}\right)\left(R_{k-1}+h^{2} B_{\delta}^{\prime} P_{k} B_{\delta}\right)^{-1} \\
& \times\left(r_{k-1}^{u}+h^{2} B_{\delta}^{\prime} r_{k}^{\gamma}+B_{\delta}^{\prime} r_{k-1}^{\delta}+h B_{\delta}^{\prime} P_{k} r_{k}^{\lambda}+h B_{\delta}^{\prime} p_{k}\right) .
\end{aligned}
$$

Hence, the search direction is calculated as follows: i) set $P_{N} \leftarrow Q_{f}$ and $p_{N} \leftarrow r_{N}^{x}$; ii) compute $P_{k}$ and $p_{k}$ for $k=N, N-1, \ldots, 2$ using the two backward recursions in (15); iii) compute $\Delta u_{0}, \Delta \gamma_{0}, \Delta \delta_{0}, \Delta \lambda_{1}$ and $\Delta x_{1}$ using (13); iv) compute, recursively, $\Delta u_{k}, \Delta \gamma_{k}, \Delta \delta_{k}$ for $k=$ $2,3, \ldots, N-1$ and $\Delta \lambda_{k}$ and $\Delta x_{k}$ for $k=1,2, \ldots, N$ using (14).

While the block reduction manipulations of [10] yielded the famous Discrete Riccati Difference Equation (DRDE) for time-varying weighting matrices [10, Eq. 51a], we obtained in (15a), analogously, a DRDE for time-varying weighting matrices in the delta domain. The reader familiar with the work of [10] will also appreciate more subtle similarities between the recursive equations of both formulations, realizing that the two methods are entirely equivalent in an infinite precision arithmetic system. It is evident, however, that the solution in delta domain requires more operations (matrix additions and multiplications) to be performed at each iteration when compared to the equivalent solution in shift domain.

\section{Algorithm properties}

Although computing the interior-point search direction requires more operations for the delta formulation, neither the computational time nor the hardware resources utilized significantly increase if care is taken in the implementation. The reason is twofold and lies in the dependencies of the operations.

Proposition 1 Let the computation of the search direction (12) be implemented in a parallel hardware architecture. Then, when compared to the shift formulation of [10]:

(i) the critical path (the longest non-parallelizable sequence of operations) remains unchanged, hence the time required to calculate the search direction (latency) is the same for both formulations;

(ii) both formulations require the same number of computational hardware resources (adders, multipliers, etc.) for implementation.

Proof. The most computationally expensive operation of the algorithm is the calculation of matrices $P_{1}, P_{2}, \ldots, P_{N-1}$ and vectors $p_{1}, p_{2}, \ldots, p_{N-1}$ using the backward recursions in (15). These have to be obtained before (13) and the forward recursions in (14) can be calculated. For the sake of simplicity, we will only consider the DRDE of (15a) and point out that the same reasoning and procedures can be applied to the whole algorithm by noticing that many intermediate results can be stored in memory and reused.

(i) Let us compare the DRDE in (15a) with the equivalent shift-domain DRDE [10]

$$
\begin{aligned}
P_{k-1}= & Q_{k-1}+A_{s}^{\prime} P_{k} A_{s}+\left(M_{k-1}+A_{s}^{\prime} P_{k} B_{s}\right) \\
& \times\left(R_{k-1}+B_{s} P_{k} A_{s}\right)^{-1}\left(M_{k-1}^{\prime}+B_{s}^{\prime} P_{k} A_{s}\right)
\end{aligned}
$$

and define matrices $\bar{A}$ and $\bar{B}$ to be $\bar{A} \triangleq h A_{\delta}$ and $\bar{B} \triangleq h B_{\delta}$ for the delta case and $\bar{A} \triangleq A_{s}$ and $\bar{B} \triangleq B_{s}$ for the shift case. The data dependencies of the calculations to compute $P_{k-1}$ for both the delta and the shift formulation are shown as a graph in Fig. 2. At a matrix level, Fig. 2 is an implementation with minimal latency, signifying that all the operations are performed as soon as the data are available (exploiting the parallelism). The seven stages required to compute the solution are determined by the sequence of operations in the critical path, which is the path on the right of both graphs indicated by thicker arrows. The critical paths for both the delta and the shift case are the same, hence the time required by both algorithms to complete is the same (although matrices $\bar{A}$ and $\bar{B}$ are different for the two cases, what matters here is their dimensions, which do not change). The search direction is computed recursively in $N$ steps in both formulations.

(ii) For the delta case, there are four extra matrix additions to be performed, shown in Fig. 2 by the boxes with dotted edges. At each stage of the algorithm, a maximum of three matrix adders and three matrix multipliers are used simultaneously. This is true for both the delta and the shift cases, implying that the same number of hardware blocks is needed for both implementations (of course, in the delta case, the adders will be used more often). If both the recursions in (15) are considered, it can be easily shown that five adders and five multipliers are needed. This is the same as for the analogous shift-domain recursions [10, Eq. 51]. Furthermore, the same adder and multiplier blocks (as well as many intermediate results) can be re-utilized efficiently for the calculation of (14).

Proposition 1 shows that the extra operations required for the delta case do not increase the algorithm latency nor the computational hardware resources utilized. Hence, the numerical advantages given by the delta implementation are obtained at no extra cost from the point of view of resource utilization and computational time. It should also be noted that for multi-input plant models $\left(n_{u}>1\right)$ the bottleneck of the algorithm is the $n_{u}$-by- $n_{u}$ matrix factorization needed in Stage 4 of Fig. 2, which is performed on matrices of the same size in both formulations. If such a factorization is obtained via a Cholesky decomposition, a number of division and square roots must be computed (much more expensive to perform than simple additions and multiplications). This motivates even further the use of a reduced number of bits 


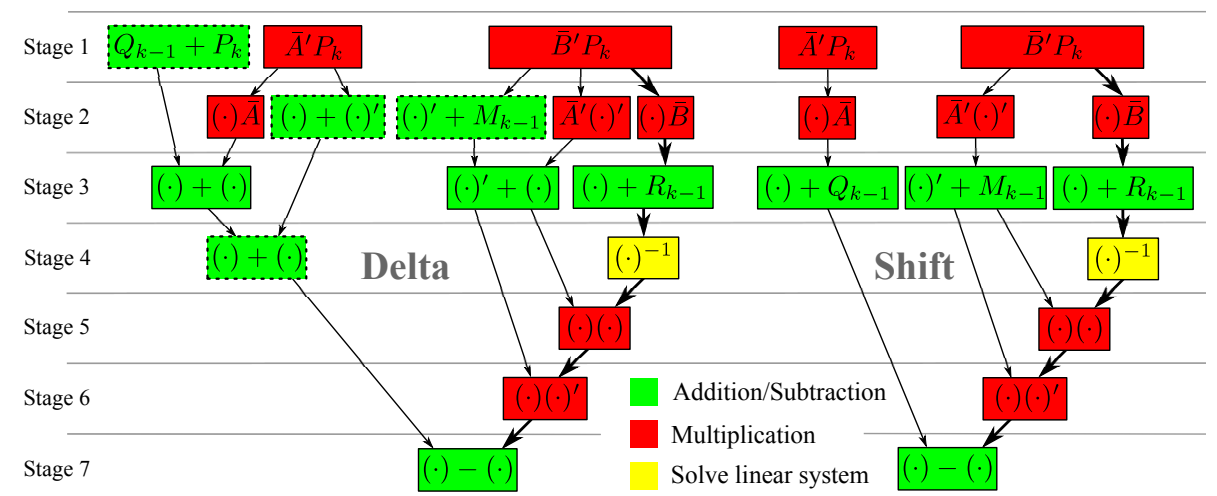

Fig. 2. Data dependencies of the delta- and shift-domain Riccati recursions. For delta, $\bar{A} \triangleq h A_{\delta}$ and $\bar{B} \triangleq h B_{\delta}$. For shift, $\bar{A} \triangleq A_{s}$ and $\bar{B} \triangleq B_{s}$. Both algorithms have the same latency because they have the same critical path (thicker arrows). At each stage, no more than three matrix additions and three matrix multiplications are performed simultaneously. Hence, although four extra additions are needed for the delta case (dotted boxes), the number of computational resources needed for minimal latency is the same in both cases.

for data representation in order to speed up the execution of division and square roots, and therefore the whole algorithm. As a consequence, an implementation of the algorithm in a sequential (rather than parallel) architecture can also have an equal latency and resource utilization if a low enough number of bits is used.

\section{Illustrative example}

In this section we show, with simulation examples, that the solution from the delta formulation is indeed more numerically accurate than the equivalent shift one; therefore, the same closed-loop performance can be achieved with our formulation using fewer bits in the number representation. Using fewer bits implies increased computational speed and reduced hardware usage. An indication of the improvement in hardware resources utilized will be given for an FPGA from the Xilinx Virtex-6 family [13]. Furthermore, simulations have shown that the increased numerical accuracy also improves the interior-point algorithm convergence speed, thus reducing the number of interior-point iterations required to completion. We use as a benchmark a spring-mass system with six unitary masses linearly connected by five springs with unitary coefficients with input forces applied to the first and last masses. The input constraints are $\left\|u_{k}\right\|_{\infty} \leq 0.5$ and the state constraints are $\left\|x_{k}\right\|_{\infty} \leq 3.5$. The associated performance measure is given by the LQ cost in (7) with matrices $Q, R, M$ and $Q_{f}$ coming from the discretization, with sampling period $h=0.01$ s, of a continuous-time LQ cost $\int_{0}^{h N} x(t)^{\prime} x(t)+u(t)^{\prime} u(t) d t$. The prediction horizon was selected as $N=200$.

First, we compare the variation of closed-loop performance when the QP solver is implemented (in floating-point) using the proposed formulation (Section 3 ) and the formulation of [10] (shift). As a performance metric we use

$$
\Gamma \triangleq \sum_{k=0}^{N_{\text {sim }}-1}\left[\begin{array}{l}
x_{k} \\
u_{k}
\end{array}\right]^{\prime}\left[\begin{array}{cc}
Q & M \\
M^{\prime} & R
\end{array}\right]\left[\begin{array}{l}
x_{k} \\
u_{k}
\end{array}\right]
$$

where the system is left to evolve from initial conditions $x_{0}=\left[\begin{array}{lllllll}0 & 0 & 3.5 & 3.5 & 0 & 0\end{array}\right]^{\prime}, u_{0}=\left[\begin{array}{ll}0 & 0\end{array}\right]^{\prime}$ and
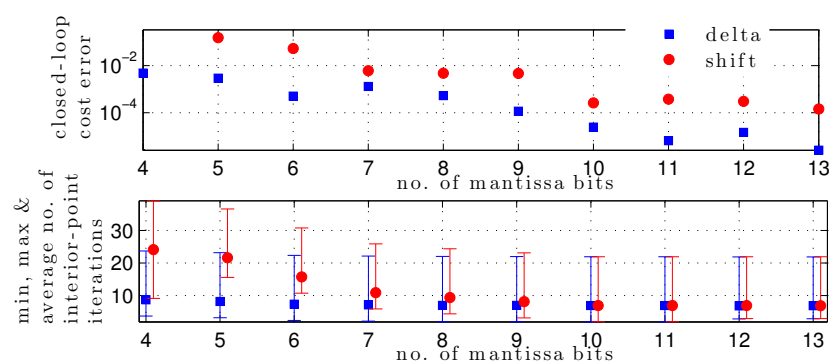

Fig. 3. For the same number of bits, the closed-loop cost error for the delta implementation is lower (top plot) as well as the number of interior-point iterations (bottom plot).

with $N_{\text {sim }}=20 / h$ (selected to be long enough to allow the system to reach steady-state). A cost, $\Gamma_{52}$, has been evaluated as in (17) for a shift implementation in double precision and for an implementation with reduced precision for data representation, which we call $\Gamma_{\text {low }}$. An error, called closed-loop cost error has been defined as $\left\|\Gamma_{\text {low }}-\Gamma_{52}\right\| /\left\|\Gamma_{52}\right\|$. The top plot of Fig. 3 shows how the closed-loop cost error varies for a range of mantissa bits for the two Riccati recursion-based implementations. This error, for the delta implementation, is always lower than for the shift implementation, especially for a small number of bits. As the number of bits grows, the difference between the two formulations becomes negligible (hence results are shown only up to 13 bits). The bottom plot of Fig. 3 shows the minimum, maximum and average number of interior-point iterations required for the duality gap to be less than $10^{-5}$ (set as a tolerance to stop the interior-point iterations). The delta formulation requires on average fewer iterations to reach the predefined threshold, implying that faster convergence can be achieved.

Fig. 4 shows some closed-loop trajectories where only a 5-bit mantissa was used for the number representation for the numerical solution of the QP. Even for such a low precision, the trajectories of the delta implementation almost completely overlap with the shift double-precision (52 bits) one. However, a traditional (shift) 5-bit implementation response becomes practically unacceptable. This is caused by numerical errors, which are primarily due to the errors in the 

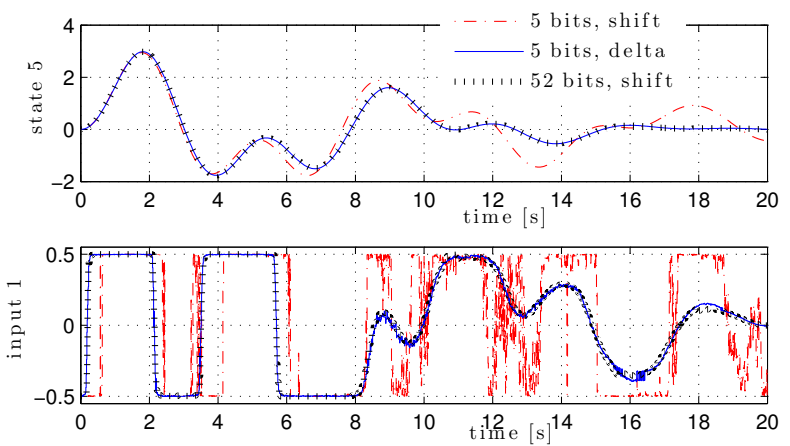

Fig. 4. While the performance of a 5-bit shift implementation is very poor, a 5-bit delta implementation produces trajectories that almost perfectly overlap the ones from a 52-bit shift implementation.
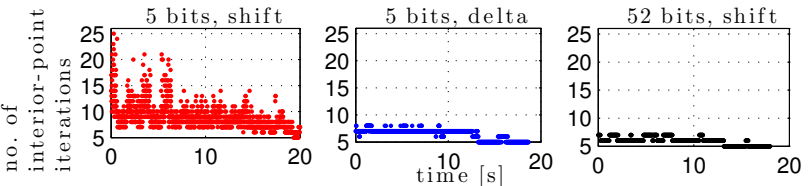

Fig. 5. Because the numerical errors in the delta formulation are lower, the number of iterations required is also lower when compared to the shift implementation.

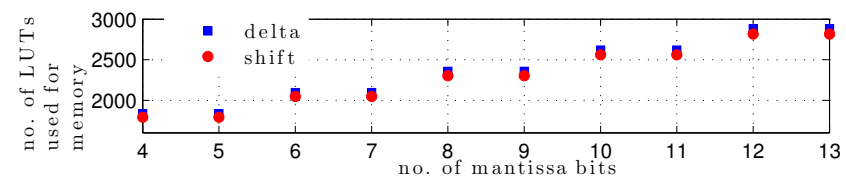

Fig. 6. The delta implementation always requires slightly more memory. However, it is sufficient to reduce the number of mantissa bits by at most two to bring the number of LUTs required by the delta implementation below the number of LUTs required by the shift implementation.

evaluation of the Riccati equation, as has also been observed in [3]. Consequently, the number of interior-point iterations is also much lower for the delta formulation, as shown in Fig. 5.

Finally, we comment on the memory resources required by each implementation. We consider an FPGA from the Virtex6 family and assume that the data words are stored in lookup tables (LUTs). We also assume that 8 bits are used for the exponent part of the floating-point numbers. Fig. 6 shows the number of LUTs required by both algorithms for different numbers of mantissa bits. The numbers of LUTs for a shift implementation in single and double precision are shown in Fig. 1. As expected, the delta implementation always requires slightly more LUTs, and hence slightly more resources. However, it is sufficient to reduce the number of mantissa bits by at most two to allow the delta implementation to utilize fewer resources than the shift one. Reducing the mantissa bits affects the closed-loop performance, but the top plot of Fig. 4 shows that a reduction of two bits still leaves the delta implementation with a better performance than the shift one.

\section{Conclusions}

We have shown that the superior numerical properties of a delta-domain formulation of a constrained LQR problem can be enjoyed without sacrificing solver complexity in terms of its execution time or computational hardware resources required. For the Riccati recursion approach to solving the linear equations in (12), the number of decision variables in the resulting QP problem is indeed larger when compared to its equivalent shift formulation. However, because of the particular data dependencies in the algorithm, the latency and arithmetic units required for the operations will not increase when care is taken in the implementation. The proposed design procedure gives the designer the flexibility to reduce the number of bits used for data representation in order to increase the computational speed and/or reduce the circuit size. This paper is a contribution toward a larger research goal to develop model predictive control algorithms with a guarantee that the loss of accuracy due to finite precision effects is minimized or bounded a priori. Future research could investigate the appropriateness of other sampled-data models and/or number representations and an extension of these ideas to the nonlinear case.

\section{References}

[1] K. Åström and B. Wittenmark. Computer-Controlled Systems Prentice-Hall, $3^{\text {rd }}$ edition, 1997.

[2] G. A. Constantinides. Tutorial paper: Parallel architectures for model predictive control. In Proc. European Control Conference 2009, pages 138-143, Budapest, HU, 2009.

[3] G. C. Goodwin, R. H. Middleton, and H. V. Poor. High-speed digital signal processing and control. Proc. IEEE, 80(2):240-259, 1992.

[4] G. C. Goodwin, J. I. Yuz, J. C. Agüero, and M. Cea. Sampling and sampled-data models. In Proc. American Control Conference, pages 1-20, Baltimore, USA, 2010

[5] E. C. Kerrigan, J. L. Jerez, S. Longo, and G. A. Constantinides. Number representation in predictive control. In Proc. IFAC Conf. on Nonlinear Model Predictive Control, volume 4, pages 60-67, Noordwijkerhout, the Netherlands, 2012.

[6] A. H. Levis, R. S. Schlueter, and M. Athans. On the behaviour of optimal linear sampled-data regulators. Int. J. Control, 13(2):343361, 1971.

[7] S. Longo, E. C. Kerrigan, and G. A. Constantinides. A predictive control solver for low-precision data representation. In Proc. European Control Conference, Zürich, Switzerland, 2013.

[8] R. H. Middleton and G. C. Goodwin. Improved finite word length characteristics in digital control using delta operators. IEEE Trans. Automatic Control, AC-31(11):1015-1021, 1986.

[9] R. H. Middleton and G. C. Goodwin. Digital Control and Estimation: A Unified Approach. Prentice-Hall, 1990.

[10] C. V. Rao, S. J. Wright, and J. B. Rawlings. Application of interiorpoint methods to model predictive control. J. Optimization Theory and Applications, 99(3):723-757, 1998.

[11] A.G. Wills, D. Bates, A.J. Fleming, B. Ninness, and S.O.R. Moheimani. Model predictive control applied to constraint handling in active noise and vibration control. IEEE Trans. Control Systems Technology, 16(1):3-12, 2008.

[12] S. J. Wright. Interior point methods for optimal control of discrete time systems. J. Optimization Theory and Applications, 77(1):161187, 1993.

[13] Xilinx. www.xilinx.com, April 2013. 